\title{
Correction to: Effect of JAK inhibitors on high- and low-density lipoprotein in patients with rheumatoid arthritis: a systematic review and network meta-analysis
}

\author{
$\mathrm{Na} \mathrm{Li}{ }^{1} \cdot$ Zhong-Ping Gou ${ }^{1}$. Shuang-Qing $\mathrm{Du}^{1} \cdot$ Xiao-Hong Zhu ${ }^{1} \cdot$ Hui Lin ${ }^{2} \cdot$ Xiu-Fang Liang ${ }^{1} \cdot$ Yong-Sheng Wang ${ }^{1}$. \\ Ping Feng ${ }^{1}$ (D)
}

Published online: 27 January 2022

(C) International League of Associations for Rheumatology (ILAR) 2022

\section{Correction to: Clinical Rheumatology https://doi.org/10.1007/s10067-021-06003-z}

In the original published version of the above article contained the following errors and should have been corrected. [Bold emphasis shows the error area]

1. The Affiliation "National Medical Products Administration Key Laboratory for Clinical Research and Evaluation of Innovative Drugs, Institute of Clinical Trials, West China Hospital, Sichuan University, Chengdu 610041, Sichuan, China" should have been "National Medical Products Administration Key Laboratory for Clinical Research and Evaluation of Innovative Drugs, Institute of Clinical Trial Center, West China Hospital, Sichuan University, Chengdu 610041, Sichuan, China"

2. Under the "Study characteristics" of "Results" section, the total number of article obtained in the text "After searching Pubmed, Medline, Embase, and Cochrane Controlled Trials Register databases a total of $\mathbf{8 0 6}$ articles was obtained." should have been 906 instead of 806. In the text, "After excluding duplicates, the title, abstract or full text of $\mathbf{4 8 8}$ articles were read to assess their eligibility according to inclusion and exclusion criteria." The correct number of articles read should have been $\mathbf{5 7 0}$ instead of $\mathbf{4 8 8}$ and the text "After excluding duplicates, the title, abstract or full text of $\mathbf{4 8 8}$ articles were read..." should be read as "After excluding duplicates, the title, abstract and full text of $\mathbf{4 8 8}$ articles were read..."

3. The Tables 2, 3, 4 and Figure 4 were not presented correctly. The corrected Tables and Figure are shown here.

The original article has been corrected.
The online version of the original article can be found at https:// doi.org/10.1007/s10067-021-06003-Z

Ping Feng

fengping@wchscu.cn; pfyq@yahoo.com

1 National Medical Products Administration Key Laboratory for Clinical Research and Evaluation of Innovative Drugs, Institute of Clinical Trial Center, West China Hospital, Sichuan University, Chengdu 610041, Sichuan, China

2 Department of Rheumatology, West China Hospital, Sichuan University, Chengdu 610041, Sichuan, China 
Table 2 Characteristics of studies included in the systematic review and network meta-analysis of the effect of JAK inhibitors on lipoprotein levels in rheumatoid arthritis (RA) patients

\begin{tabular}{|c|c|c|c|c|c|c|c|}
\hline Study & Study type & $\begin{array}{l}\text { Study drug } \\
\text { (no. of patients) }\end{array}$ & $\begin{array}{l}\text { female } \\
\text { patients(n\%) }\end{array}$ & Age (years) & RA duration (years) & Baseline DAS28 & $\begin{array}{l}\text { Follow-up } \\
\text { (weeks) }\end{array}$ \\
\hline \multirow[t]{2}{*}{ Fleischmann et al. 2012} & \multirow[t]{2}{*}{ Phase IIb } & $\begin{array}{l}\text { Tofacitinib } \\
\text { (49) }\end{array}$ & 87.8 & $54 \pm 13.5$ & $13.7 \pm 8.4$ & 5.6 & 24 \\
\hline & & $\begin{array}{l}\text { Placebo } \\
\text { (59) }\end{array}$ & 88.1 & $53 \pm 13.7$ & $16.6 \pm 10.4$ & 5.6 & 24 \\
\hline \multirow[t]{2}{*}{ Kremer et al. 2012} & \multirow[t]{2}{*}{ Phase IIb } & $\begin{array}{l}\text { Tofacitinib } \\
\text { (71) }\end{array}$ & 80.3 & $52 \pm 12.8$ & $16.1 \pm 9.6$ & 5.1 & 24 \\
\hline & & $\begin{array}{l}\text { Placebo } \\
\text { (69) }\end{array}$ & 81.2 & $53 \pm 13.4$ & $14.5 \pm 8.1$ & 5.3 & 24 \\
\hline \multirow[t]{2}{*}{ Kremer et al. 2009} & \multirow[t]{2}{*}{ Phase IIa } & $\begin{array}{l}\text { Tofacitinib } \\
\text { (61) }\end{array}$ & 86.9 & $47.9 \pm 10.8$ & 10.2 & 6.2 & 6 \\
\hline & & $\begin{array}{l}\text { Placebo } \\
(65)\end{array}$ & 84.6 & $51.3 \pm 12.1$ & 8.7 & 6.0 & 6 \\
\hline \multirow[t]{2}{*}{ Fleischmann et al. 2017} & \multirow[t]{2}{*}{ Phase III } & $\begin{array}{l}\text { Baricitinib } \\
(215)\end{array}$ & 73 & $49 \pm 14$ & $1.3 \pm 2.7$ & 5.9 & 52 \\
\hline & & $\begin{array}{l}\text { Placebo } \\
(210)\end{array}$ & 70 & $51 \pm 13$ & $1.3 \pm 4$ & 5.9 & 52 \\
\hline \multirow[t]{2}{*}{ Taylor et al. 2017} & \multirow[t]{2}{*}{ Phase III } & $\begin{array}{l}\text { Baricitinib } \\
(487)\end{array}$ & 77 & $54 \pm 2$ & $10 \pm 9$ & 5.8 & 24 \\
\hline & & $\begin{array}{l}\text { Placebo } \\
(488)\end{array}$ & 78 & $53 \pm 2$ & $10 \pm 9$ & 5.7 & 24 \\
\hline \multirow[t]{2}{*}{ Dougados et al. 2016} & \multirow[t]{2}{*}{ Phase III } & $\begin{array}{l}\text { Baricitinib } \\
(227)\end{array}$ & 82 & $52 \pm 12$ & $8 \pm 8$ & 5.6 & 24 \\
\hline & & $\begin{array}{l}\text { Placebo } \\
(228)\end{array}$ & 83 & $51 \pm 13$ & $7 \pm 8$ & 5.5 & 24 \\
\hline \multirow[t]{2}{*}{ Genovese et al. 2016} & \multirow[t]{2}{*}{ Phase III } & $\begin{array}{l}\text { Baricitinib } \\
(177)\end{array}$ & 84 & $56 \pm 11$ & $14 \pm 9$ & 5.9 & 24 \\
\hline & & $\begin{array}{l}\text { Placebo } \\
(176)\end{array}$ & 82 & $56 \pm 11$ & $14 \pm 10$ & 5.9 & 24 \\
\hline \multirow[t]{2}{*}{ Tanaka et al. 2016} & \multirow[t]{2}{*}{ Phase IIb } & $\begin{array}{l}\text { Baricitinib } \\
(24)\end{array}$ & 79 & $57.5 \pm 10.4$ & $5.9 \pm 4$ & 4.96 & 12 \\
\hline & & $\begin{array}{l}\text { Placebo } \\
(49)\end{array}$ & 80 & $51.1 \pm 12$ & $5.1 \pm 4$ & 4.72 & 12 \\
\hline \multirow[t]{2}{*}{ Keystone et al. 2014} & \multirow[t]{2}{*}{ Phase IIb } & $\begin{array}{l}\text { Baricitinib } \\
(52)\end{array}$ & 71 & $53 \pm 10$ & $5.3 \pm 4.5$ & 5.3 & 12 \\
\hline & & $\begin{array}{l}\text { Placebo } \\
(98)\end{array}$ & 87 & $49 \pm 12$ & $5.4 \pm 4.3$ & 5.5 & 12 \\
\hline \multirow[t]{2}{*}{ Takeuchi et al. 2019} & \multirow[t]{2}{*}{ Phase III } & $\begin{array}{l}\text { Peficitinib } \\
(174)\end{array}$ & 71.8 & $56.2 \pm 11.6$ & $4.4 \pm 3.1$ & 5.4 & 12 \\
\hline & & $\begin{array}{l}\text { Placebo } \\
\text { (170) }\end{array}$ & 71.2 & $55.3 \pm 12.1$ & $4.3 \pm 2.9$ & 5.4 & 12 \\
\hline \multirow[t]{2}{*}{ Tanaka et al. 2019} & \multirow[t]{2}{*}{ Phase III } & $\begin{array}{l}\text { Peficitinib } \\
(102)\end{array}$ & 76.5 & $55 \pm 12.8$ & $10.4 \pm 8.2$ & 5.4 & 12 \\
\hline & & $\begin{array}{l}\text { Placebo } \\
\text { (101) }\end{array}$ & 72.3 & $56.3 \pm 11.7$ & $7 \pm 6.6$ & 5.4 & 12 \\
\hline \multirow[t]{2}{*}{ Genovese et al. 2017} & \multirow[t]{2}{*}{ Phase IIb } & $\begin{array}{l}\text { Peficitinib } \\
\text { (64) }\end{array}$ & 78.1 & $54.4 \pm 12.5$ & $10.5 \pm 8.2$ & 5.9 & 12 \\
\hline & & $\begin{array}{l}\text { Placebo } \\
(51)\end{array}$ & 82.4 & $52.7 \pm 12.2$ & $9.8 \pm 7.7$ & 5.9 & 12 \\
\hline \multirow[t]{2}{*}{ Kivitz et al. 2016} & \multirow[t]{2}{*}{ Phase IIb } & $\begin{array}{l}\text { Peficitinib } \\
\text { (78) }\end{array}$ & 82.1 & $54.2 \pm 12.5$ & $7.3 \pm 6.3$ & 5.6 & 12 \\
\hline & & $\begin{array}{l}\text { Placebo } \\
\text { (72) }\end{array}$ & 87.5 & $52.6 \pm 12.2$ & $7.2 \pm 7.7$ & 5.4 & 12 \\
\hline
\end{tabular}


Table 2 (continued)

\begin{tabular}{|c|c|c|c|c|c|c|c|}
\hline Study & Study type & $\begin{array}{l}\text { Study drug } \\
\text { (no. of patients) }\end{array}$ & $\begin{array}{l}\text { female } \\
\text { patients(n\%) }\end{array}$ & Age (years) & RA duration (years) & Baseline DAS28 & $\begin{array}{l}\text { Follow-up } \\
\text { (weeks) }\end{array}$ \\
\hline \multirow[t]{2}{*}{ Takeuchi et al.2015 } & Phase IIb & $\begin{array}{l}\text { Peficitinib } \\
\text { (58) }\end{array}$ & 88 & $51.6 \pm 12.1$ & $7 \pm 5.2$ & 5.4 & 12 \\
\hline & & $\begin{array}{l}\text { Placebo } \\
(56)\end{array}$ & 77 & $54.2 \pm 12.1$ & $6.9 \pm 5.4$ & 5.1 & 12 \\
\hline \multirow[t]{2}{*}{ Combe et al. 2021} & phase III & $\begin{array}{l}\text { Filgotinib } \\
(480)\end{array}$ & 83.1 & $53 \pm 12.6$ & $8.5 \pm 8.2$ & 5.7 & 24 \\
\hline & & $\begin{array}{l}\text { Placebo } \\
(475)\end{array}$ & 82.3 & $53 \pm 12.8$ & $7.3 \pm 7.2$ & 5.7 & 24 \\
\hline \multirow[t]{2}{*}{ Kavanaugh et al. 2016} & Phase IIb & $\begin{array}{l}\text { Filgotinib } \\
(70)\end{array}$ & 75.7 & $53 \pm 1.4$ & $9 \pm 0.8$ & 6.2 & 12 \\
\hline & & $\begin{array}{l}\text { Placebo } \\
\text { (72) }\end{array}$ & 77.8 & $52.0 \pm 1.4$ & $10.0 \pm 0.8$ & 6.2 & 12 \\
\hline \multirow[t]{2}{*}{ Rubbert-Roth et al. 2020} & Phase III & $\begin{array}{l}\text { Upadacitinib } \\
\text { (303) }\end{array}$ & 82 & $55.3 \pm 11.4$ & $12.4 \pm 9.5$ & 5.7 & 24 \\
\hline & & $\begin{array}{l}\text { Abatacept } \\
(309)\end{array}$ & 82 & $55.8 \pm 11.9$ & $11.8 \pm 8.3$ & 5.9 & 24 \\
\hline \multirow[t]{2}{*}{ Fleischmann et al. 2019} & Phase III & $\begin{array}{l}\text { Upadacitinib } \\
(651)\end{array}$ & 80 & $54 \pm 12$ & $8 \pm 8$ & 5.8 & 14 \\
\hline & & $\begin{array}{l}\text { Placebo } \\
(651)\end{array}$ & 79 & $54 \pm 12$ & $8 \pm 8$ & 5.8 & 14 \\
\hline
\end{tabular}

Dosage of JAK inhibitors: tofacitinib, $5 \mathrm{mg}$ twice daily; baricitinib, $4 \mathrm{mg}$ once daily; upadacitinib, $15 \mathrm{mg}$ once daily; peficitinib, $150 \mathrm{mg}$ once daily; filgotinib, $100 \mathrm{mg}$ once daily. DMARDS means disease modifying antirheumatic drug; DAS28, disease activity score in 28 joints; NA, no data available

Table 3 League table: network meta-analysis results comparing the effects of JAK inhibitors on high-density lipoprotein levels (mg/dl)

\begin{tabular}{lllllll}
\hline & Baricitinib & & & & & \\
Filgotinib & $1.67(-4.94,8.22)$ & Filgotinib & & & \\
Peficitinib & $-2.86(-7.86,1.97)$ & $-4.53(-11.35,2.15)$ & Peficitinib & & \\
Placebo & $7.75(4.41,11.10)$ & $6.09(0.40,11.77)$ & $10.61(7.08,14.30)$ & Placebo & & \\
Tofacitinib & $1.24(-5.75,8.29)$ & $-0.40(-8.74,7.95)$ & $4.11(-2.95,11.31)$ & $-6.51(-12.68,-0.34)$ & Tofacitinib & \\
Upadacitinib & $0.50(-7.87,8.82)$ & $-1.17(-10.71,8.38)$ & $3.35(-5.04,11.88)$ & $-7.26(-14.91,0.37)$ & $-0.75(-10.64,9.02)$ & Upadacitinib \\
\hline
\end{tabular}

The value in the box indicates the mean difference (95\% CI) between the drug listed horizontally and the drug listed vertically 
Table 4 League table: network meta-analysis results comparing the effects of JAK inhibitors on low-density lipoprotein levels (mg/dl)

Baricitinib

Filgotinib $\quad 5.052(-4.67,14.33) \quad$ Filgotinib

Peficitinib $\quad 7.03(-0.17,13.79) \quad 1.96(-7.79,11.67) \quad$ Peficitinib

Placebo $\quad 13.53(8.72,18.19) \quad 8.47(0.37,16.92) \quad 6.50(1.52,11.79) \quad$ Placebo

Tofacitinib $\quad-0.07(-11.88,11.51) \quad-5.12(-18.60,8.47) \quad-7.06(-18.95,4.78) \quad-13.59(-24.38,-3.02) \quad$ Tofacitinib

Upadacitinib $\quad-8.24(-19.88,3.28) \quad-13.33(-26.49,0.33) \quad-15.30(-26.81,-3.37) \quad-21.78(-32.28,-11.20) \quad-8.21(-23.03,6.91) \quad$ Upadacitinib

The value in the box indicates the mean difference $(95 \% \mathrm{CI})$ between the drug listed horizontally and the drug listed vertically 


\section{Treatment Control}

Study

Events Total Events Total

Tofacitinib

Fleischmann et al. 2012

Kremer et al.2012

Kremer et al.2009

Fixed effect model

Random effects model

Heterogeneity: not applicable

\section{Baricitinib}

Fleischmann et al. 2017

Taylor et al.2017

Dougados et al.2016

Genovese et al.2016

Tanaka et al.2016

Keystone et al. 2014

Fixed effect model

Random effects model

Heterogeneity: $I^{2}=18 \%, \tau^{2}=0.5297, p=0.30$

\section{Peficitinib}

Tanaka et al.2019

Takeuchi et al. 2019

Genovese et al.2017

Kivitz et al.2017

Takeuchi et al.2015

Fixed effect model

$\begin{array}{rrrr}0 & 49 & 0 & 59 \\ 0 & 71 & 0 & 69 \\ 0 & 61 & 0 & 65 \\ & \mathbf{1 8 1} & & \mathbf{1 9 3}\end{array}$

Random effects model

$\begin{array}{rrrr}2 & 215 & 0 & 210 \\ 0 & 487 & 1 & 488 \\ 2 & 227 & 0 & 228 \\ 0 & 177 & 2 & 176 \\ 0 & 24 & 0 & 49 \\ 0 & 52 & 0 & 98 \\ & 1182 & & 1249\end{array}$

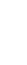

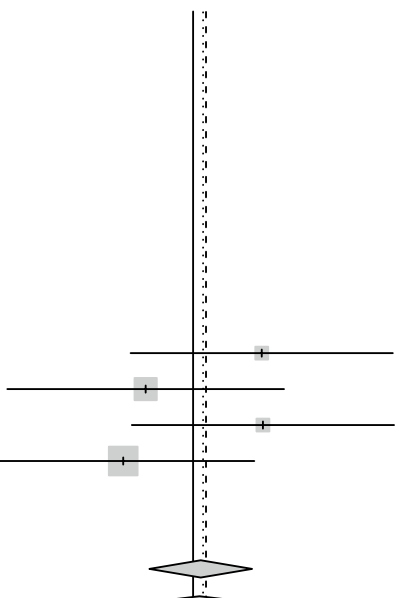

RR

Weight Weight

Risk Ratio

95\%-Cl

$\begin{array}{rrrr}0 & 102 & 0 & 101 \\ 1 & 174 & 1 & 170 \\ 0 & 64 & 1 & 51 \\ 0 & 78 & 0 & 72 \\ 0 & 58 & 0 & 56 \\ & \mathbf{4 7 6} & & \mathbf{4 5 0}\end{array}$

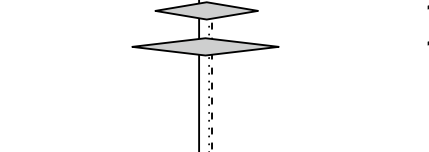

$4.88 \quad[0.24 ; 101.13] \quad 4.7 \%$

$9.7 \%$

$0.33 \quad[0.01 ; 8.18] \quad 14.0 \%$

$8.7 \%$

$5.02[0.24 ; 104.03] \quad 4.7 \%$

$0.20 \quad[0.01 ; 4.11] \quad 23.4 \%$

$9.7 \%$

$9.7 \%$

$0.0 \%$

$1.19[0.36 ; 3.90] \quad 46.9 \%$

$0.0 \%$

$1.16[0.21 ; 6.30] \quad-$

$37.6 \%$
$0.0 \% \quad 0.0 \%$

$0.98 \quad[0.06 ; 15.49] \quad 9.5 \% \quad 11.6 \%$

$0.27 \quad[0.01 ; 6.40] \quad 15.6 \% \quad 8.8 \%$

$0.0 \% \quad 0.0 \%$

$0.0 \% \quad 0.0 \%$

$0.53[0.07 ; 3.90] \quad 25.0 \%$

$0.56[0.07 ; 4.49] \quad-$

$20.4 \%$

Heterogeneity: $\iota^{2}=0 \%, \tau^{2}=0, p=0.54$

Upadacitinib

$\begin{array}{lllll}\text { Rubbert-Roth et al.2020 } & 1 & 303 & 0 & 309\end{array}$

$\begin{array}{lllll}\text { Fleischmann et al.2019 } & 3 & 651 & 0 & 651\end{array}$

Fixed effect model $\quad 954 \quad 960$

Random effects model

Heterogeneity: $I^{2}=0 \%, \tau^{2}=0, p=0.71$

\section{Filgotinib}

Combe et al. 2021

Kavanaugh et al.2016

Fixed effect model

Random effects model

Heterogeneity: not applicable

Fixed effect model

Random effects model

Heterogeneity: $I^{2}=0 \%, \tau^{2}=0, p=0.62$

$\begin{array}{rrrr}2 & 480 & 2 & 475 \\ 0 & 70 & 0 & 72 \\ & \mathbf{5 5 0} & & \mathbf{5 4 7}\end{array}$

547

Test for subgroup differences (fixed effect): $\chi_{3}^{2}=2.38, \mathrm{df}=3(p=0.50) \quad 0.01$

Test for subgroup differences (random effects): $\chi_{3}^{2}=2.10, \mathrm{df}=3(p=0.55)$
3399

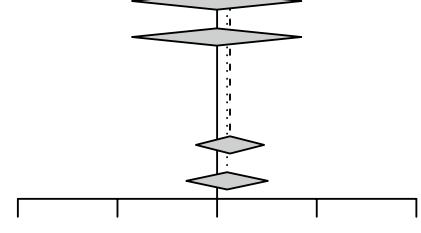

$0.1 \quad 1 \quad 10 \quad 100$

Fig. 4 Pooled estimates of relative risk of major cardiovascular events in rheumatoid arthritis patients, stratifed by JAK inhibitor treatment

Publisher's note Springer Nature remains neutral with regard to

jurisdictional claims in published maps and institutional affiliations. 